\title{
Glottopol
}

Revue de sociolinguistique en ligne

$33 \mid 2020$

Varia - Liberté, égalité, diversité !

\section{Présentation : liberté, égalité, diversité !}

\section{Clara Mortamet}

\section{OpenEdition}

Journals

Édition électronique

URL : https://journals.openedition.org/glottopol/523

DOI : 10.4000/glottopol.523

ISSN : 1769-7425

\section{Éditeur}

Presses universitaires de Rouen et du Havre

Référence électronique

Clara Mortamet, «Présentation : liberté, égalité, diversité ! », Glottopol [En ligne], 33 | 2020, mis en ligne le 01 janvier 2020, consulté le 02 octobre 2021. URL : http://journals.openedition.org/glottopol/523 ; DOI : https://doi.org/10.4000/glottopol.523 


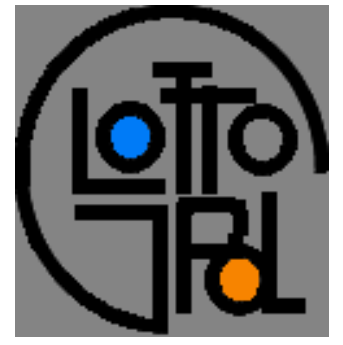

\section{GLOTTOPOL}

Revue de sociolinguistique en ligne n`33 - février 2020

Varia : Liberté, égalité, diversité !

\section{SOMMAIRE}

Hommages à Jean-Baptiste Marcellesi : À ringrazià vi, par Niculau Sorba ; "Ciao Ziu ! » par Philippe Blanchet.

Clara Mortamet : Présentation du numéro : Liberté, égalité, diversité !

Manon Him-Aquilli : "Prendre la parole sans prendre le pouvoir ». La mise en registre de l'horizontalité dans les assemblées générales anarchistes/autonomes.

Siham Hocini : La formulation du désaccord dans les débats télévisés algériens : quels "effetsde-politesse "?

Pauline Rannou: Trajectoires de la surdité en France. Parents entendants d'enfants sourds: questionner la fragmentation de l'identité sourde.

Catherine Combaz-Champlaine: Les rectifications orthographiques de 1990 comme révélateurs du rapport des enseignants à l'orthographe.

Marie-Madeleine Bertucci : Éléments d'épistémologie pour l'étude des espaces ségrégués des banlieues urbaines sensibles de la région parisienne.

Élise Gandon: Usages du numérique et illectronisme: nouvelles tâches pour le lecteurscripteur, nouveaux enjeux d'apprentissage.

Marc Arabyan : Quelle langue pour la recherche neurolinguistique française?

José R. de Arellano : Le rapprochement entre l'espagnol et le portugais pendant la décennie dorée sud-américaine : une problématique de l'horizontalité supercentrale.

Adam Wilson: Normes interactionnelles globalisées et communautés de pratique discontinues : les dynamiques sociolinguistiques du tourisme international.

Mélanie Lancien : Caractérisation de la variation liée à la situation de communication : apport de l'acoustique à la phonostylistique.

Assémou Maurice Ludovic Assémou : La prononciation du français parlé en Côte d'Ivoire.

http://glottopol.univ-rouen.fr 


\title{
PRÉSENTATION : LIBERTÉ, ÉGALITÉ, DIVERSITÉ !
}

\author{
Clara Mortamet \\ Université de Saint Etienne
}

\section{Tout un hommage}

Ce numéro 33 de la revue Glottopol est le premier Varia. Le nombre de textes reçus montre $_{1}$ à lui seul, si besoin en était, l'utilité de cet espace de publication ouvert à partir et autour des questionnements posés dans les années 1980 par des sociolinguistes rouennais. Quelques mois après la disparition de Jean-Baptiste Marcellesi, nous voulons voir dans ce succès un signe de ce que les concepts qu'il a forgés s'avèrent fertiles bien au-delà de son existence, et s'inscrivent à présent dans le temps long de la vie des idées. Ce numéro publie les hommages de Niculau Sorba et de Philippe Blanchet, mais lui rend hommage aussi à travers la diversité des terrains et des objets explorés. Les textes réunis rendent compte, chacun et ensemble, des interrogations qui traversent la discipline et au-delà les sociétés francophones. Il nous ramène à des questions sans cesse reposées, celles du vivre ensemble et de la hiérarchie de nos valeurs, que renouvelle aujourd'hui la mondialisation.

L'expérience d'un numéro Varia fut motivée au départ par le souci de donner une opportunité de publication à des recherches marginales, émergentes ou décalées, qui trouvent peu de place dans les numéros thématiques habituels. Le numéro qui parait révèle finalement des objets d'étude revisités plus qu'inédits. Signes de l'évolution de nos sociétés, de notre histoire contemporaine, ils s'inscrivent dans une certaine continuité avec ce qui les a précédés, tout en pointant des besoins ou des perspectives nouveaux. Ainsi l'illectronisme prolonge la question de l'illettrisme ; l'étude des assemblées générales anarchistes renouvelle l'étude de la parole contestataire et des modes d'interaction ; le débat télévisé apparait comme une version moderne des joutes oratoires ; la réforme de l'orthographe vue par les enseignants 30 ans après son adoption revisite les premiers travaux sur cette question; la question des banlieues des décennies après leur embrasement en France permet de faire le point de la situation; la description de la variation linguistique, diatopique ou diastratique continue de nourrir une

\footnotetext{
${ }_{1}$ Je ne reviendrai pas ici sur le processus éditorial particulier que suppose un numéro Varia. Je signale seulement que nous avons reçu 28 textes, que nous en avons envoyé 24 en expertise à 41 relecteurs (!), et qu'il en est resté finalement 11. La plupart des avis positifs étaient assortis de très nombreuses demandes de modification; certains textes ont fait l'objet d'avis contradictoires, arbitrés chaque fois par un troisième expert. Dans l'ensemble un numéro Varia oblige à se poser davantage la question des frontières de la sociolinguistique francophone, de ce qui fait un article, une démarche, un terrain, une problématisation dans notre discipline. L'expérience a laissé des questions en suspens, et a probablement frustré plus d'un auteur et d'un relecteur. Elle a eu pour mérite d'interroger, de croiser des avis et perspectives entre relecteurs, et entre auteurs et relecteurs.
} 
analyse des relations entre les locuteurs et entre les communautés linguistiques; le refus des assignations identitaires prolonge les travaux sur les minorités et les minorisations ; l'étude des choix de langues par les locuteurs revisite leurs rapports et places respectifs. Chaque fois, les questions posées sont à la fois anciennes et actuelles, témoignent de ce qu'elles demeurent en suspens, appellent à des états des lieux ou à de nouvelles approches théoriques.

\section{Liberté, égalité, diversité}

Les 11 textes réunis ici disent à leur façon quelque chose de l'état de notre discipline, mais peut-être aussi de l'état de notre monde et de nos préoccupations. Il m'est rapidement apparu qu'ils posaient - ou plutôt je l'ai dit qu'ils reposaient - la question des valeurs qui nous animent quand il s'agit de vivre ensemble à l'heure de la mondialisation. J'y ai lu trois aspirations : la liberté, l'égalité, et la diversité ; mais j'y ai lu aussi les tensions et conflits que produit l'attachement simultané à ces trois valeurs.

La liberté dont il est question dans ce numéro est d'une part celle de la parole, qui permet à chacun d'exprimer son point de vue, de participer à la vie publique. On la trouve dans les articles de Manon Him-Aquili, qui étudie les conflits de gestion de la parole dans les assemblées générales anarchistes, ainsi que dans le texte de Siham Hocini, qui s'intéresse à l'expression des désaccords dans des émissions de débat télévisées. La liberté est aussi celle d'appartenances plurielles, en diachronie comme en synchronie, à travers la question de l'identité des enfants sourds de parents entendants (Pauline Rannou). Il est encore question de la liberté d'appliquer, ou de ne pas appliquer la réforme de l'orthographe (Catherine Combaz).

L'égalité est présente dans le texte de Marie-Madeleine Bertucci qui renouvelle le constat de l'exclusion des populations assignées aux banlieues, et fait le point sur ce que cette exclusion continue de dire de notre organisation collective et de l'inégale jouissance des libertés individuelles. L'égalité dont il est question est aussi celle des locuteurs face aux usages des langues, comme dans le cas des situations d'illectronisme (Elise Gandon). C'est aussi l'égalité des langues les unes par rapport aux autres, que ce soit au sein d'une communauté de parole particulière - l'usage majoritaire de l'anglais dans certaines publications scientifique (Marc Arabyan) -, à l'échelle d'un sous-continent - l'espagnol et le portugais en Amérique latine (José R. de Arellano) ou encore au sein de l' «espace monde » qu'est une office du tourisme à Marseille (Adam Wilson). Cette question de l'égalité rappelle à certains la nécessité de préserver, cultiver, mettre en valeur la diversité des langues, de leurs usages, et de leurs locuteurs. Si cette préoccupation pour la diversité pointe déjà à travers la quête d'égalité et de liberté, elle est aussi abordée à travers les études sur la variation. Les usages hétérogènes du français ont ceci de précieux qu'ils témoignent des relations interpersonnelles et des types de discours (Mélanie Lancien), ou bien d'appropriations du français en francophonie (la Côte d'Ivoire d'Assémou Maurice Ludovic Assémou).

Bien que dominantes dans l'un ou l'autre des textes réunis, les aspirations de liberté, d'égalité et de diversité traversent en réalité presque tous les textes, et y apparaissent le plus souvent en tension les unes avec les autres. Ainsi la difficulté est ainsi moins de s'accorder sur leur importance ou leur priorité que sur leur difficile articulation.

\section{Débattre dans la mondialisation}

Certains textes convoquent alors des notions ou perspectives visant à renouveler la façon de penser ces contradictions. Cela justifie des approches parfois assez théoriques, autorise quelques auteurs à sortir un peu du modèle de l'article scientifique. Cela permet aussi de faire 
le point sur une situation et d'expliciter d'où l'on parle, pour s'autoriser aussi à dire à quelle sociolinguistique on aspire, dans quelle direction on s'engage.

Certaines notions et objets traversent enfin des textes aux terrains souvent très différents. On notera ainsi par exemple les notions d'horizontalité (Manon Him-Aquilli, José R. de Arellano) et de communautés de pratiques (Adam Wilson, Marc Arabyan); les objets que sont la variation (Assémou Maurice Ludovic Assémou, Mélanie Lancien), les identités plurielles (MarieMadeleine Bertucci, Pauline Rannou), les interactions (Adam Wilson, Siham Hocini, Mélanie Lancien et Manon Him-Aquilli), et les normes d'écriture (Élise Gandon, Catherine Combaz, Marc Arabyan).

On trouve enfin, et peut-être surtout, la nécessité d'inventer des lieux et des formats, de faire surgir des sujets et des occasions du débat, d'augmenter nos capacités à exposer nos idées de façon contradictoire, tout en préservant la liberté de chacun à y participer. L'attention portée dans ce numéro à la co-existence des opinions, des points de vue et des pratiques variées, que l'on cherche moins à réduire qu'à faire cohabiter, signe ainsi l'aspiration à créer, dans un monde mondialisé, un vivre ensemble qui entretienne la diversité des langues, des identités, des subjectivités et des situations de parole. 


\section{GLOTTOPOL}

Revue de sociolinguistique en ligne

Comité de rédaction : Michaël Abecassis, Salih Akin, Sophie Babault, Claude Caitucoli, Véronique Castellotti, Régine Delamotte, Robert Fournier, Stéphanie Galligani, Emmanuelle Huver, Normand Labrie, Foued Laroussi, Benoit Leblanc, Fabienne Leconte, Gudrun Ledegen, Danièle Moore, Clara Mortamet, Alioune Ndao, Isabelle Pierozak, Gisèle Prignitz.

Rédactrice en chef : Clara Mortamet.

Comité scientifique : Claudine Bavoux, Michel Beniamino, Jacqueline Billiez, Philippe Blanchet, Pierre Bouchard, Ahmed Boukous, Pierre Dumont, Jean-Michel Eloy, Françoise Gadet, Monica Heller, Caroline Juilliard, Jean-Marie Klinkenberg, Jean Le Du, Marinette Matthey, Jacques Maurais, Marie-Louise Moreau, Robert Nicolaï, Didier de Robillard, Paul Siblot, Claude Truchot, Daniel Véronique.

\section{Comité de lecture pour ce numéro :}

Mickael Abecassis, Michelle Auzanneau, Salih Akin, Céline Amourette, Gabriel Bergounioux, Philippe Boula de Mareuil, Catherine Brissaud, Aude Bretegnier, Maria Candea, Véronique Castellotti, Régine Delamotte, Jean-François De Pietro, Catherine Delarue-Breton, Anne Dister, Alexandre Duchêne, Valentin Feussi, Françoise Gadet, Stéphanie Galligani, François Gaudin, Monica Heller, Emmanuelle Huver, Caroline Juilliard, Abou Bakri Kebe, Jean-Marie Klinkenberg, Christian Lagarde, Foued Laroussi, Fabienne Leconte, Evelyne Lloze, Nolwenn Lorenzi, Gudrun Ledegen, Nadja Maillard, Marinette Matthey, Bruno Maurer, Véronique Miguel Addisu, Saskia Mugnier, Claire Oger, Marielle Rispail, Richard Sabria, Véronique Traverso, Christel Troncy, Cécile Van den Avenne. 